\title{
Problematización y conocimiento especializado del formador de profesores de Matemáticas
}

\author{
Hugo Parra-Sandoval \\ hugoparras@hdes.luz.edu.ve \\ https://orcid.org/0000-0002-4484-2619 \\ Universidad del Zulia (LUZ) \\ Maracaibo, Venezuela
}

Recibido: 29/04/2020 Aceptado: 20/05/2020

\begin{abstract}
Resumen
Se presenta una reflexión sobre el lugar que ocupa la problematización en el conocimiento del formador de profesores de matemáticas. Tanto la problematización de saberes como los estudios sobre el formador de profesores son temas que se han trabajado escasamente y mucho menos se ha visto cómo ellos se pueden articular para mejorar los procesos de formación de profesores de matemáticas. La problematización es un término bastante utilizado en la formación de docentes; sin embargo, poco se ha profundizado sobre su naturaleza y desarrollo en los procesos formativos; de igual manera, los avances en el tema del conocimiento del formador de profesores no han sido tampoco muy trabajados, a pesar de la importancia que se le reconoce a este actor en los procesos de transformación educativa. Para abordar la relación entre la problematización y el conocimiento especializado del profesor en los procesos de formación, desarrollamos algunas ideas sobre los tipos de conocimientos que un formador de profesores de matemáticas debería tener; luego se aclara lo que se entiende por problematización, su caracterización y las condiciones para que ésta se desarrolle en los procesos formativos. Finalmente se reconoce que son los espacios formativos donde la problematización y el conocimiento del formador de profesores se complementan para lograr procesos formativos caracterizados por la reflexión sobre la práctica. Concluimos con una serie de ideas de lo que podría ser una agenda de investigación sobre estos dos aspectos: el de la problematización y el conocimiento del formador de profesores
\end{abstract}

Palabras claves: Formador de Profesores, Problematización, Conocimiento del profesor

\section{Problematização e conhecimento especializado do professor de Matemática}

\section{Resumo}

É apresentada uma reflexão sobre o lugar que a problematização ocupa no conhecimento do formador de professores de matemática. Tanto a problematização do conhecimento quanto os estudos sobre o professor formador são assuntos pouco trabalhados, muito menos visto como eles podem ser articulados para melhorar os processos de formação de professores de matemática. Problematização é um termo amplamente utilizado na formação de professores; no entanto, pouco foi feito sobre sua natureza e desenvolvimento nos processos de treinamento; Da mesma forma, os avanços no tema do conhecimento do professor formador também não foram muito trabalhados, apesar da importância que esse ator é reconhecido nos processos de transformação educacional. Para abordar a relação entre problematização e conhecimento especializado de professores em processos de treinamento, algumas idéias são desenvolvidas sobre os tipos de conhecimento que um professor de matemática deve ter; Em seguida, esclarece-se o que se entende por problematização, sua caracterização e as condições para que 
ele se desenvolva nos processos de treinamento. Por fim, reconhece-se que são os espaços de formação em que a problematização e o conhecimento do professor formador se complementam para alcançar processos de formação caracterizados pela reflexão sobre a prática. Conclui com uma série de idéias do que poderia ser uma agenda de pesquisa sobre esses dois aspectos: o da problematização e o conhecimento do professor formador

Palavras chaves: Formador de Professores, Problematização, Conhecimento do professor

\title{
Problematization and specialized knowledge of the Mathematics Teacher's Educator
}

\begin{abstract}
A reflection is presented on the place that problematization occupies in the knowledge of the mathematics teacher trainer. Both the problematization of knowledge and studies on the teacher educator are subjects that have been scarcely worked on, much less seen how they can be articulated to improve the training processes of mathematics teachers. Problematization is a term widely used in teacher training; however, little has been done about its nature and development in the training processes; in the same way, the advances in the subject of the knowledge of the teacher educator have not been much worked either, in spite of the importance that this actor is recognized in the processes of educational transformation. To address the relationship between problematization and specialized teacher knowledge in training processes, some ideas are developed about the types of knowledge that a mathematics teacher educator should have; Then it is clarified what is meant by problematization, its characterization and the conditions for it to develop in training processes. Finally, it is recognized that they are the training spaces where the problematization and the knowledge of the teacher educator complement each other to achieve training processes characterized by reflection on practice. It concludes with a series of ideas of what could be a research agenda on these two aspects: that of problematization and the knowledge of the teacher educator
\end{abstract}

Keywords: Teacher Educator, Problematization, Teacher knowledge

\section{Introducción}

El presente escrito aborda la problematización de los saberes como modo de proceder del formador de profesores de matemáticas. Aunque el formador de profesores es considerado un actor clave de la calidad educativa, las investigaciones sobre la conformación de su conocimiento profesional y su actuación han sido escasas. Una revisión de las publicaciones que abordan el tema del formador de profesores en tres de las revistas de Latinoamérica dedicadas a la Educación Matemática presentes en Scopus, muestra que entre los años 2014 y 2019 sólo fueron reseñados ocho trabajos. Esta cantidad representa tan solo un 6,4\% en relación al total de artículos de formación de profesores y el 1,7\% del total de trabajos publicados en esas mismas revistas (Ver tabla 1) 
Tabla 1.

Artículos relacionados con la formación de profesores y formadores de profesores. (2014-2019)

\begin{tabular}{|c|c|c|c|c|c|c|}
\hline \multirow[t]{2}{*}{ Revista } & \multirow[t]{2}{*}{$\begin{array}{l}\text { Total } \\
\text { artículos }\end{array}$} & \multicolumn{2}{|c|}{$\begin{array}{l}\text { Artículos de } \\
\text { formación de } \\
\text { profesores }\end{array}$} & \multicolumn{3}{|c|}{ Artículos del formador de profesores } \\
\hline & & $\begin{array}{l}\text { Número } \\
\text { artículos }\end{array}$ & $\begin{array}{c}\% \text { Respecto } \\
\text { al total }\end{array}$ & $\begin{array}{l}\text { Número } \\
\text { artículos }\end{array}$ & $\begin{array}{l}\text { \% respecto al } \\
\text { total de art }\end{array}$ & $\begin{array}{c}\text { \% respecto al } \\
\text { total art de } \\
\text { formación } \\
\text { de } \\
\text { profesores }\end{array}$ \\
\hline BOLEMA & 311 & 102 & $32,8 \%$ & 6 & $1,93 \%$ & 5,88 \\
\hline $\begin{array}{l}\text { Educación } \\
\text { Matemática }\end{array}$ & 99 & 14 & $14,14 \%$ & 1 & $1,01 \%$ & $7,14 \%$ \\
\hline RELIME & 60 & 9 & $15 \%$ & 1 & $1,66 \%$ & $11,11 \%$ \\
\hline Total & 470 & 125 & $26,6 \%$ & 8 & $1,7 \%$ & $6,4 \%$ \\
\hline
\end{tabular}

Fuente: elaborado por el autor

Esta poca presencia en los trabajos publicados contrasta con lo afirmado en diferentes documentos institucionales (UNESCO, 2016). En ellos se manifiesta que el formador de profesores es un actor clave, por ser él uno de los principales responsables del diseño e implementación de los programas de formación y su labor incide directa e indirectamente en la calidad educativa (González-Vallejo, 2018; UNESCO, 2016; Vaillant y Marcelo, 2015)).

Aunque se ha estudiado muy poco al formador de profesores, no se puede decir lo mismo del profesor de los niveles preuniversitario (Educación inicial, primaria y media). Desde la década de los ochenta Shulman (1986) formuló su tesis en la que señalaba que el conocimiento del profesor estaba conformado por tres grandes dominios: conocimiento del contenido a enseñar, conocimiento didáctico del contenido y conocimiento curricular. A partir de entonces surgió un interés cada vez más creciente por investigar al profesor y éste no ha dejado de crecer. Un ejemplo del interés por estudiar al profesor ha sido la Educación Matemática; en los últimos quince años se han venido desarrollando diferentes modelos que buscan representar el conocimiento del profesor de matemáticas. Ejemplos de ellos encontramos en el modelo denominado knowledge quartet (KQ) desarrollado por Rowland, T., Huckstep, P., \& Thwaites (2005). De igual manera, Ball y sus colaboradores han presentado su modelo denominado MKT (Mathematical Knowledge for Teaching) (Ball \& al., 2008; Hill \& al., 2008; Ball \& Bass, 2009). 
También, hallamos el modelo del "Conocimiento Especializado del Profesor" o MTSK por sus siglas en inglés, desarrollado por un equipo de profesores de la universidad de Huelva en España bajo el liderazgo del profesor José Carrillo (Carrillo, Climent, Contreras y Muñoz-Catalán, 2013) o, el modelo denominado Conocimientos y Competencias Didáctico-Matemáticos (CCDM) desarrollado por Godino, Giacomone, Font y Pino-Fan (2018) que deriva del enfoque Ontosemiótico creado originalmente por un equipo liderado por el profesor Juan Godino. Todos estos modelos comparten su interés por el estudio del profesor de matemáticas y todos tomaron como referencia de origen a Shulman (1986). Estos modelos, han fijado particularmente su atención en dos de los dominios presentados por Shulman: el Conocimiento del Contenido a enseñar y el Conocimiento Pedagógico del Contenido.

Estos modelos que abordan el conocimiento del profesor de matemáticas en general son insuficientes para explicar el conocimiento profesional del formador de profesores. Hay la convicción de que el conocimiento del formador de profesores incluye otros conocimientos, además de los contemplados para el profesor de matemáticas en general (Chick and Beswick, 2017; Sánchez y García, 2004). Entre aquellos otros conocimientos que debe tener el formador de profesores, Sánchez y García (2004) hacen referencia a uno que particularmente nos interesa para el tema que deseamos abordar, se trata del conocimiento que el formador de profesores debe tener para poder "crear situaciones en las que se pueda producir una reflexión sobre los procesos de aprender a enseñar matemáticas" (Sánchez y García, 2004: p. 486).

Esta creación de espacios y momentos para la reflexión están acompañados por la problematización de situaciones relacionadas con los proceso de enseñanza y aprendizaje de las matemáticas. Aun cuando la problematización, es un término bastante común en la literatura de las Ciencias Sociales, poco se ha discutido acerca de su naturaleza (Fabre, 2005). No obstante, la problematización de la práctica de los profesores es un elemento clave en el desarrollo de procesos formativos caracterizados por la reflexión (Borjas, Ortiz, Rodríguez, Soto, 2015; Shön, 1998)

Ante la poca investigación sobre el formador de profesores y las posibilidades que ofrece la problematización para generar procesos formativos caracterizados por la reflexión sobre la práctica, cabe preguntarse cuál será el lugar de la problematización en el marco del conocimiento del formador de profesores de matemáticas. Para responder a esta interrogante abordamos en primer lugar algunas premisas sobre los conocimientos que un formador de 
profesores de matemáticas debería tener; luego pasamos a aclarar lo que entendemos por problematización, su caracterización y las condiciones para que ésta se desarrolle en los procesos formativos. Finalmente, abordamos el lugar que ocupa la problematización en el conocimiento del formador de profesores.

\section{Conocimiento Profesional del Formador de Profesores}

Tres décadas después de que Shulman (1986) planteara que el conocimiento del profesor tiene sus propias características, han surgido diferentes modelos que buscan representarlo. En el caso del profesor de matemáticas citábamos los modelos de Rowland et al. (2005), Ball et al (2008), Carrillo y col. (2013) y Godino y col. (2017). Sin embargo, con el transcurrir del tiempo los estudios han hecho evidente que dentro de esta comunidad los formadores de estos profesores, no están del todo bien representados en los modelos mencionados.

Una primera aproximación para establecer la diferencia entre el conocimiento del profesor de matemáticas y el profesor que los forma, podría pensarse en términos de cantidad de conocimiento; es decir, que el formador de profesores debe saber más que el profesor que ha formado o está formando. Sin embargo, esta idea es muy vaga ¿Qué significa "saber más que el profesor a quien ha formado o está formando? Este planteamiento reduce la diferencia a un problema de acumulación de conocimientos, lo cual no es suficiente. La diferencia no es solo de cantidad; la diferencia abarca también la incorporación de otros tipos de conocimientos propios de la acción del profesor que forma a otros profesores; por esa razón, si bien es necesario que un formador de profesores de matemáticas haya sido o sea un buen profesor de matemáticas en los niveles pre-universitarios, esto no es suficiente. El formador de profesores debe poseer otros conocimientos que lo diferencian del buen profesor de matemáticas (Chick \& Bewuick, 2017; Sánchez y García, 2004).

En la idea de buscar modelos o referentes que puedan caracterizar el conocimiento del formador, Chick y Beswuick (2017) han planteado que el formador de profesores de matemática debe tener un conocimiento pedagógico del contenido diferente al conocimiento pedagógico del contenido del profesor de los niveles pre-universitarios; de alguna manera éste debe poseer un meta-conocimiento pedagógico del contenido. En ese sentido plantean que el formador de profesores posee básicamente tres tipos o dominios de conocimientos: 
- Conocimiento pedagógico del contenido de las matemáticas escolares

- Conocimiento del contenido matemático en contextos pedagógicos

- Conocimiento pedagógico en un contexto matemático

Entre los tres tipos de conocimientos Chick y Beswick (2017) plantean veintidós categorías, de las cuales retomaremos más adelante algunas para explicar la presencia de la problematización en el conocimiento del formador de profesores.

El conocimiento pedagógico del contenido de las matemáticas escolares en el formador de profesores permite plantear situaciones donde sus estudiantes puedan ver claramente la relación estrecha que debe haber entre el conocimiento matemático y cómo éste se aprende y se enseña. Por ejemplo, en el momento que un estudiante para profesor responda de manera errada, el formador de profesores debe tener la capacidad de hacerle ver el error a través de ejemplos, contraejemplos o preguntas, de manera que se percate de su falla; pero esta toma de conciencia por parte del futuro profesor debe estar acompañada de una reflexión acerca de las causas que pudieron haber producido el error y cómo este error debe ser tratado en caso de que el futuro profesor se le presente una situación semejante al momento de ejercer de su profesión.

Un segundo tipo de conocimiento es el que Chick y Beswick llaman conocimiento del contenido matemático en contextos pedagógicos. El formador de profesores deberá identificar aspectos críticos del conocimiento del contenido, como por ejemplo, la demanda cognitiva que una nueva operación representa para los estudiantes y los errores más comunes asociados a esta.

Por último Chick y Beswick (2017) presentan lo que ellos llaman conocimiento pedagógico en un contexto matemático. Se trata de hacer uso de conocimientos pedagógicos generales como la planificación de una clase o la evaluación de un tema y contextualizarlos en un ambiente de enseñanza y aprendizaje de las matemáticas; pudiera ser el caso de una propuesta de evaluación que hay que analizar estableciendo si está bien o no formulada de acuerdo a criterios didácticos matemáticos.

En grandes rasgos, estos tres tipos de conocimientos permitirían al formador de profesores de matemática trabajar con sus participantes diferentes situaciones propias del contexto de la enseñanza y aprendizaje de las matemáticas, en los niveles donde ejercerá la profesión docente.

\section{Problematización}


Tal y como ya señalamos, el término "problematización” es y ha sido muy utilizado en la literatura de las ciencias sociales; Kosik (1967) en la década de los años sesenta del siglo pasado hacía mención al término en el campo de la filosofía, cuando realizó un estudio sobre la cotidianidad. Para esa misma época, Freire (1970) lo incorporó con fuerza en el campo de la educación crítica. Luego D’Ambrosio en la década de los años setenta del siglo XX incorporó el término en las primeras publicaciones que se hicieron sobre la etnomatemática (Peña-Rincón, Hueitra-Santibañez, 2016). De la misma manera, Skovsmose y Valero (2007), Cantoral, ReyesGasperini y Montiel (2015) y Baez y Farfán (2017) entre otros, han venido incorporando el término en la literatura de nuestra disciplina.

Sin embargo, aunque el término "problematización” ha sido bastante mencionado, pocas veces se aclara su significado preciso. Al respecto Fabre (2005) señala que "Hoy, en la enseñanza y la formación de adultos, la idea del problema, la problemática y la problematización se convoca masivamente como si estas nociones fueran evidentes" (p. 1, traducción propia). A pesar de que es común dejar por sobreentendido el término problematización, autores como Rodríguez (2015), Bless y Diaz (2010), Musquer (2009), Diaz (2008), Foucault (1984) y Dewey (1920, citado por Fabre, 2006) han trabajado su conceptualización desde perspectivas diferentes; aunque en su mayoría, desde los contextos de formación.

Al adentrarse en el significado de la problematización de estos autores, hallamos que Foucault (1984) plantea la problematización como un conjunto de prácticas, discursivas o no, que hace al individuo preguntarse qué es lo verdadero o lo falso de una situación. Para Focault, la problematización no es la representación de un objeto inexistente sino un objeto cognoscible, relacionado con la reflexión que hace el sujeto que se plantea el dilema entre lo que percibe como cierto y lo que percibe como falso. Esta asunción de dilemas genera datos y condiciones (Fabre y Musquer, 2009) que, al relacionarse con las experiencias de quienes problematizan, generan procesos de reflexión importantes (Diaz, 2008). En el caso de la formación de docentes, la problematización se nutre de dos fuentes; por una parte, en la formación inicial el futuro profesor establece una relación con las experiencias de su vida estudiantil y por otra, en el caso de los profesores que ya están ejerciendo su profesión, se le une a su experiencia de vida, como estudiante, las experiencias derivadas propiamente de su práctica como docente. Al tener su origen en la experiencia, la problematización adquiere un sentido histórico en los sujetos que la 
practican, carácter que Freire (2002) ya lo señalaba y que Bless y Diaz (2010) lo reafirman cuando indican que "la problematización es una actividad orientada al cuestionamiento de lo que damos por cierto. Se trata de una actividad que busca develar el origen histórico-cultural de los conceptos que creamos para nombrar fenómenos y de las prácticas que inventamos para manejarlos" (p. 12).

La problematización de los saberes constituye una actividad clave en cualquier proceso formativo de los profesores; ella busca cuestionar lo aparentemente cierto, creando en los sujetos situaciones dilemáticas que generan reflexión a partir de datos y condiciones que surgen de la misma situación, lo que se enriquece con las experiencias de vida de estos mismos sujetos involucrados en la actividad.

\section{Características de la Problematización}

Fabre (2005), quien ha trabajado la problematización en el contexto del aula, señala cinco características: la primera es que es un proceso multidimensional que implica posicionamiento, construcción y resolución por parte de los sujetos ante la situación planteada. Veamos el siguiente ejemplo de una sesión formativa de maestros en ejercicio, donde se discutían diferentes maneras de enseñar las fracciones. Una maestra afirmó lo siguiente:

"Yo por ejemplo, a veces, cuando quería representar un quinto, algunos niños dividían el círculo en cuatro partes iguales y luego agarraban una de las partes y le pasaban una rayita por el medio y se supone que una fracción debe ser dividida en cinco partes iguales...en ese aspecto se debe sugerir (al niño) que se debe seleccionar la figura adecuada y decir cuales figuras geométricas se pueden dividir en partes iguales y cuáles no"

El grupo de docentes que escuchaba quedó en silencio sin objetar lo dicho por la docente. En ese momento el facilitador intervino diciendo:

“Quiere decir que un círculo no puede ser dividido en cinco partes iguales?...”

Y la maestra asintiendo, respondió:

Si, así es

El facilitador, dirigiéndose al resto de los participantes preguntó:

¿Los demás qué opinan? ¿Están de acuerdo?

A partir de estas dos últimas preguntas hubo posicionamiento por parte de los participantes porque se desencadenaron una serie de opiniones a favor y en contra, cada una con sus respectivas argumentaciones. Con el desarrollo de las opiniones se fue construyendo un 
consenso en torno a la inexactitud de lo afirmado por la maestra y de esta manera, se llegó a una resolución de la problemática planteada.

La segunda característica de un proceso de problematización es que va de una situación desconocida a una conocida; en el episodio reseñado, el facilitador creó -a través de las interrogantes- una duda, un problema (lo desconocido) y en el transcurso del intercambio de ideas por parte de todos los participantes se buscó resolver la situación, hasta llegar a concluir que lo afirmado inicialmente era matemáticamente incorrecto (lo conocido). Pero esta transición de lo "desconocido" a lo "conocido" no se da de manera lineal y automática, sino a través de una dialéctica entre los hechos, la experiencia de los participantes y la o las teorías que se ponen en juego en este proceso; esta dialéctica es la tercera característica de la problematización; sin ella, no hay reflexión.

La cuarta característica señalada por Fabre es la presencia de un pensamiento controlado por normas (intelectuales, éticas, técnicas, pragmáticas...), siendo estas normas algunas veces predefinidas y otras construidas; En el caso de la formación de profesores hay ambos tipos de normas implícitas y explícitas; por momentos hay lo que se llama un contrato didáctico que implícitamente le otorga al formador un rol dominante, por más que éste dé muestra de confianza y libertad para que se puedan expresar. En el ejemplo citado el hecho de que el facilitador o formador formulara una pregunta inmediatamente después de la errónea afirmación, marcó una ruta en la reflexión de los participantes. Si no hubiese intervenido el facilitador, lo más probable es que la ruta de la sesión habría sido diferente, bien porque todos hubiesen aceptado lo afirmado por la maestra o porque otro participante lo cuestionara. En cualquier caso, la intervención del facilitador o el de los participantes implicarían rutas diferentes porque ambos difieren en su rol y la normativa implícita o explícita define el camino a seguir. También se puede ver la presencia de estas normas desde la mirada de los participantes; muchos de ellos, seguramente la mayoría, tenían claro desde la teoría, que la fracción un quinto si podía ser representada a través de un círculo y su discurso trató de atenerse a esta "normativa" que las matemáticas establecen.

La quinta y última característica se refiere a la existencia de una esquematización funcional de la realidad por parte del individuo que está problematizando, de manera que va construyendo herramientas para pensar y actuar sobre el hecho cuestionado. Fabre (2005) aludiendo a Dewey indica que una persona ante una situación problemática debe asumir una posición y ésta la hace desde un punto de vista funcional. En el caso de los participantes, ellos 
van tomando datos de la discusión generada por las preguntas formuladas por el facilitador y en función de ellas, van interviniendo porque ven que la solución al dilema va por el camino que pauta el intercambio de ideas. Es en función de las intervenciones que los participantes van asumiendo posiciones.

\section{Dispositivos Generadores de la Problematización}

La problematización de saberes no es producto del azar, al contrario, es intencional. La problematización es un proceso complejo que está conformado por un conjunto de operaciones que buscan determinar datos y condiciones y generar una serie de hipótesis que puedan ser corroboradas por esos mismos aspectos. La problematización tiene características procesuales no lineales, sino heurísticas, es un ir y venir de los datos y de las condiciones a las hipótesis formuladas (Dewey, 1993). Este proceso se da si hay la presencia de ciertas condiciones que permitan una conducción adecuada del proceso (Fabre y Musquer, 2009).

Borjas y colaboradores (2015) hacen mención a la presencia de dispositivos que permitan deconstruir y reconstruir los saberes de los participantes; Fabre y Musquer (2009), por su parte, indican que en todo proceso de problematización existen una serie de "inductores" que la posibilitan. Sin embargo, tal y como lo advierten estos autores, la idea de inductor no está supeditada a una relación "estímulo-respuesta" porque resultaría contradictorio con el característico ir y venir de este proceso. Cuando Fabre y Musquer hacen mención a la existencia de unos inductores, se refieren a un conjunto de acciones discursivas, o no, que orientan la reflexión y que están relacionadas con los procesos de interacción entre el formador y los participantes. Al estar relacionado con las interacciones entre los actores del proceso, la problematización dependerá en gran parte de la habilidad del formador de profesores para que estas interacciones generen procesos de cuestionamiento y movilicen sus saberes. La ausencia o no de una pregunta, la emergencia de un ejemplo o contraejemplo adecuado a los intereses y necesidades de los participantes por parte del formador de profesores, podría movilizar, o no, los saberes de los participantes. Regresemos al episodio formativo de las maestras y maestros descrito anteriormente. Cuando la maestra afirma que no puede representarse en un círculo una fracción que tenga como denominador al número cinco, el facilitador aprovecha la circunstancia y no la deja pasar por alto ya que sabe que podría haber algunos participantes que piensen lo mismo o, no tienen la certeza de si tal afirmación es o no correcta. 
Esa capacidad de captar la oportunidad para lograr efectos formativos a través de su problematización dio origen a que el formador planteara a la docente una interrogante que, posteriormente, él mismo redirige a los demás participantes. Estas acciones del facilitador, junto a otras interrogantes, ejemplos y contraejemplos que promueven la reflexión entre los docentes, es lo que Fabre y Musquer (2009) denomina inductores.

\section{Los Procesos Formativos como Lugar de Encuentro entre el Formador de Profesores y la Problematización}

Los procesos formativos son por excelencia el espacio de trabajo privilegiado para el formador de profesores. En estos espacios de interacción, los participantes se nutren de los conocimientos del formador y viceversa.

En los procesos verdaderamente formativos hace su aparición la reflexión, aspecto clave para el aprendizaje (Zaslavsky y Leikin, 1999), pero esta reflexión debe estar sustentada en el análisis de las prácticas escolares (Chapman, 2011) y este análisis viene acompañado de su problematización.

La reflexión en y sobre las prácticas escolares debe ser el resultado de la problematización guiada por el formador de profesores, aunque hay que resaltar que las intervenciones de los diferentes participantes contribuyen en esta empresa. El formador tiene la responsabilidad de que sus estudiantes descubran sus conocimientos previos sobre el tema, amplíen sus conocimientos y los confronten con sus pares, con otros autores con el saber matemático y el saber didáctico matemático (Borjas y col., 2015: p. 106; Llinares, 2013).

En estos procesos, el formador de profesores debe configurar un entorno de aprendizaje que posibilite la reflexión, seleccionando, diseñando y secuenciando tareas matemáticamente relevantes para el aprendizaje de sus estudiantes (Ivars, Fernández, Llinares, 2016; Llinares, 2013); de esta manera los futuros docentes podrán dotar de significado todos los conceptos e ideas relacionados con los procesos de enseñanza y aprendizaje de las matemáticas.

En este proceso de dotar de significado los procesos de enseñanza y aprendizaje de las matemáticas, el formador deberá poner en juego sus conocimientos que, de acuerdo a Chick y Beswick (2017), conforman su conocimiento pedagógico del contenido como formador de profesores. Recordemos que eran tres los conocimientos esenciales que identificaban al formador de profesores: conocimiento pedagógico del contenido de las matemáticas escolares; conocimiento del contenido matemático en contextos pedagógicos y conocimiento pedagógico 
en un contexto matemático. De esos tres dominios del conocimiento, proponían veintidós categorías. En lo que sigue se considerarán los tres dominios del conocimiento planteados por Chick y Bewick (2017), pero solo se tomarán y adaptarán siete categorías porque ellas responden directamente a la problematización (ver Cuadro2)

Cuadro 2.

Dominios del Conocimiento del Formador de Profesores

\begin{tabular}{|l|l|}
\hline \multicolumn{1}{|c|}{ CONOCIMIENTO } & \multicolumn{1}{|c|}{ CATEGORÍAS } \\
\hline $\begin{array}{l}\text { Conocimiento Pedagógico del contenido de } \\
\text { las matemáticas escolar }\end{array}$ & $\begin{array}{l}\text { Conocimiento de situaciones } \\
\text { ejemplares } \\
\text { Conocimientos erróneos del } \\
\text { estudiante para profesor }\end{array}$ \\
\hline $\begin{array}{l}\text { Conocimiento del contenido matemático en } \\
\text { contextos pedagógicos }\end{array}$ & $\begin{array}{l}\text { Comprensión profunda del contenido } \\
\text { fundamental } \\
\text { Deconstrucción del contenido en sus } \\
\text { componentes claves } \\
\text { Estructuras y conexiones }\end{array}$ \\
\hline $\begin{array}{l}\text { Conocimiento pedagógico en un contexto } \\
\text { matemático }\end{array}$ & $\begin{array}{l}\text { Enfoques de la evaluación } \\
\text { Diseño de actividades }\end{array}$ \\
\hline
\end{tabular}

Fuente: elaborado por el autor

\section{La experiencia compartida como punto de encuentro entre la problematización y} el conocimiento pedagógico del contenido de las matemáticas escolares

Tanto el formador de profesores como sus estudiantes comparten vivencias relacionadas con los procesos de enseñanza y aprendizaje de las matemáticas, bien como estudiantes o como profesores. Esta experiencia común es el punto de encuentro entre el formador de profesores y los futuros docentes o docentes en ejercicio; pero a la vez, es el punto de partida de todo proceso de problematización de los saberes del profesor (Bless y Díaz, 2010; Freire, 2002). Está de parte del formador identificar esas experiencias de manera que resulten significativas para los participantes.

La selección por parte del formador no es una tarea sencilla, es una de las características de su conocimiento especializado (Chick y Beswick, 2017; Sánchez y García, 2004). No es un conocimiento propio del profesor de matemáticas de los niveles pre-universitarios, ni tampoco es un conocimiento que deba tener un matemático.

El formador de profesores debe poseer una amplia gama de conocimientos de situaciones ejemplares que puedan ser motivo de interés para sus estudiantes. Esta capacidad de identificarlas es parte de lo que Chick y Beswick (2017) llaman Conocimiento Pedagógico del 
Contenido de las Matemáticas Escolares y que en este caso se ubicaron en la categoría de conocimiento de situaciones ejemplares. Veamos un ejemplo:

En una sesión formativa donde el tema era la enseñanza de las fracciones, se les pidió a los participantes que dieran su opinión respecto a la siguiente situación de clase:

Una maestra escribe en el pizarrón el siguiente ejercicio:

Dadas las siguientes fracciones representarlas gráficamente

$$
\frac{1}{4}, \quad \frac{2}{5}, \quad \frac{4}{9}
$$

Al finalizar la maestra recogió las hojas y un niño respondió de la siguiente manera

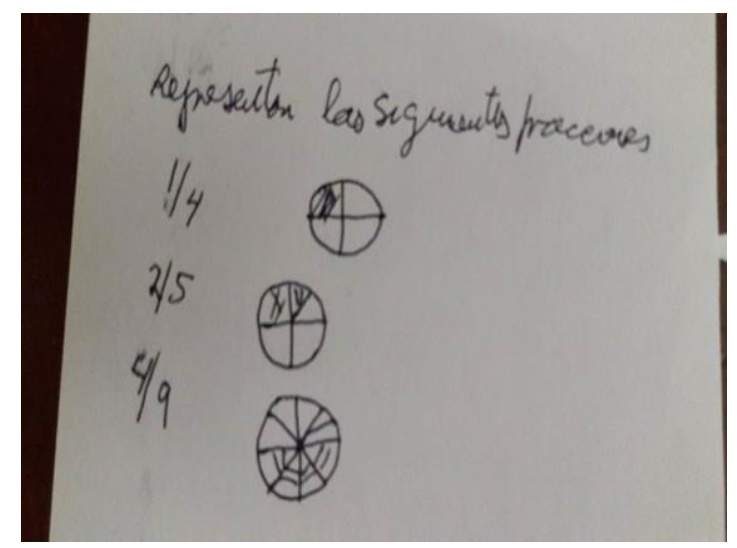

¿Qué opinas acerca de las respuestas de este niño?

El formador pide a los participantes que en un lapso de unos diez minutos, de manera individual, escriban sus argumentos para luego ser compartidos en una plenaria. Finalizado el tiempo de trabajo individual, el formador abre el derecho de palabra y comienza el intercambio de ideas.

En la experiencia registrada se evidencia que esta tarea captó la atención de los participantes desde el primer momento. La respuesta del niño con sus respectivos errores es muy común cuando se inician en la representación gráfica de las fracciones. La selección no fue hecha al azar. Se sabía por un diagnóstico previo realizado en las escuelas participantes, que este era un error que se repetía entre los niños y niñas y se seleccionó este ejemplo por considerarlo significativo desde el punto de vista educativo matemático. 
Otro aspecto que se dio en este episodio y se comentó anteriormente es la afirmación de la maestra, quien manifestaba que un quinto como fracción no podía ser representado en un círculo. En el episodio el formador de esa sesión logró captar el error de la maestra y lo trabajó de tal manera que mediante una serie de preguntas y contraejemplos pudo lograr que la maestra y aquellos que probablemente pensaban igual pero que no lo manifestaron, pudieran caer en conciencia del error y rectificar. El formador manejó pedagógicamente el contenido con sus participantes.

\section{El conocimiento del contenido matemático a enseñar en el contexto escolar}

Uno de los conocimientos especializados de formador de profesores es el conocer los diferentes temas o contenidos que se trabajan en el ámbito escolar. Este conocimiento no es solo nominal, es decir, no se trata solamente de identificar la lista de contenidos matemáticos establecidos en los currículos escolares; el formador de profesores debe poseer una comprensión profunda de esos contenidos, de sus componentes, de la estructura y sub- estructura a la cual pertenece y a sus conexiones intra y extra matemáticas.

Consideremos el mismo episodio de la respuesta del niño a la tarea que le asignó la maestra. El formador de profesores debe tener muy claro que cuando nos referimos a una fracción en el contexto escolar hablamos de una cantidad relativa, en la que su naturaleza es diferente a la de un número entero. Debe tener claro que una fracción es la expresión de la relación entre una parte y un todo, donde el todo es considerado como unidad y que la fracción puede ser planteada de diferentes maneras: como una relación parte-todo, como razón, como división de dos cantidades enteras o como un número racional. Igualmente, debe tener claro que la fracción en la escuela está en conexión con otros temas ya trabajados en grados anteriores o en conexión con contenidos de los grados que le siguen. También debe estar claro que las fracciones están relacionadas a experiencias que se viven fuera de la escuela y que por ser conocidas por los niños deben ser tomadas en cuenta, nos referimos por ejemplo a términos y procedimientos como "la mitad de..." o "un cuarto de..." que son utilizados muchas veces en gestiones de compra-venta o actividades de reparto; estas actividades extraescolares pueden variar en cuanto a sus niveles de formalidad, pero son igualmente importantes aunque muchas veces no se les valore desde la institución escolar (Parra-Sandoval y Villa-Ochoa, 2017). Todo este conocimiento y muchos más debe poseerlo el formador de profesores. 
Al ser conocedor del tema matemático escolar en profundidad, en el ejemplo citado: la representación gráfica de las fracciones, el formador de profesores está en la capacidad de aprovechar el ejemplo y adentrarse en los componentes esenciales de dicho concepto, conectando la representación de las fracciones con otros conceptos matemáticos, como la equivalencia de fracciones o la expresión decimal de un número fraccionario. Todo esto sin embargo no se haría de manera expositiva magistral, sino que se plantearía a través de la problematización de saberes matemáticos, preguntando, formulando contra ejemplos, de manera que determinadas certezas se cuestionen y permitan generar procesos reflexivos que lleven a los estudiantes para profesor o a los docentes en ejercicio a cuestionar concepciones erróneas o a reafirmar conocimientos verdaderos desde el punto de vista matemático. La problematización posibilitaría la captación de la esencia y fundamentos de los conceptos relacionados con la representación gráfica de las fracciones. Este proceso de problematización estaría enmarcado en las tres categorías relativas al conocimiento del contenido matemático en contextos pedagógicos, en particular a los que hacen mención las categorías comprensión profunda del contenido fundamental, deconstrucción del contenido en sus componentes claves y estructuras y conexiones planteados por Chick y Beswick (2017).

\section{Lo pedagógico visto en el contexto matemático escolar}

El conocimiento pedagógico en muchos países no goza de mayor prestigio, probablemente porque se redujo a una serie de indicaciones técnicas, vacías de contenido que no responden a las necesidades de las didácticas específicas, como la didáctica de las matemáticas. Sin embargo, no se trata de descartar el pensamiento pedagógico, lo que se busca es un replanteamiento de lo pedagógico a la luz de las necesidades formativas de los que se están formando para ser profesores.

En el caso que nos concierne se propone que el formador de profesores conozca de aspectos pedagógicos, como por ejemplo: la planificación y la evaluación, pero contextualizados a los procesos de enseñanza y aprendizaje de las matemáticas. El formador de profesores deberá conocer de ellos y aplicarlos adecuadamente atendiendo las necesidades e intereses de los que se están formando.

En una actividad con profesores de Matemáticas de Educación Media se les presentó un ejemplo de una evaluación planteada en una institución escolar. El formador quería con ella evaluar el conocimiento colocando como ejemplo una prueba escrita (ver figura 1) 


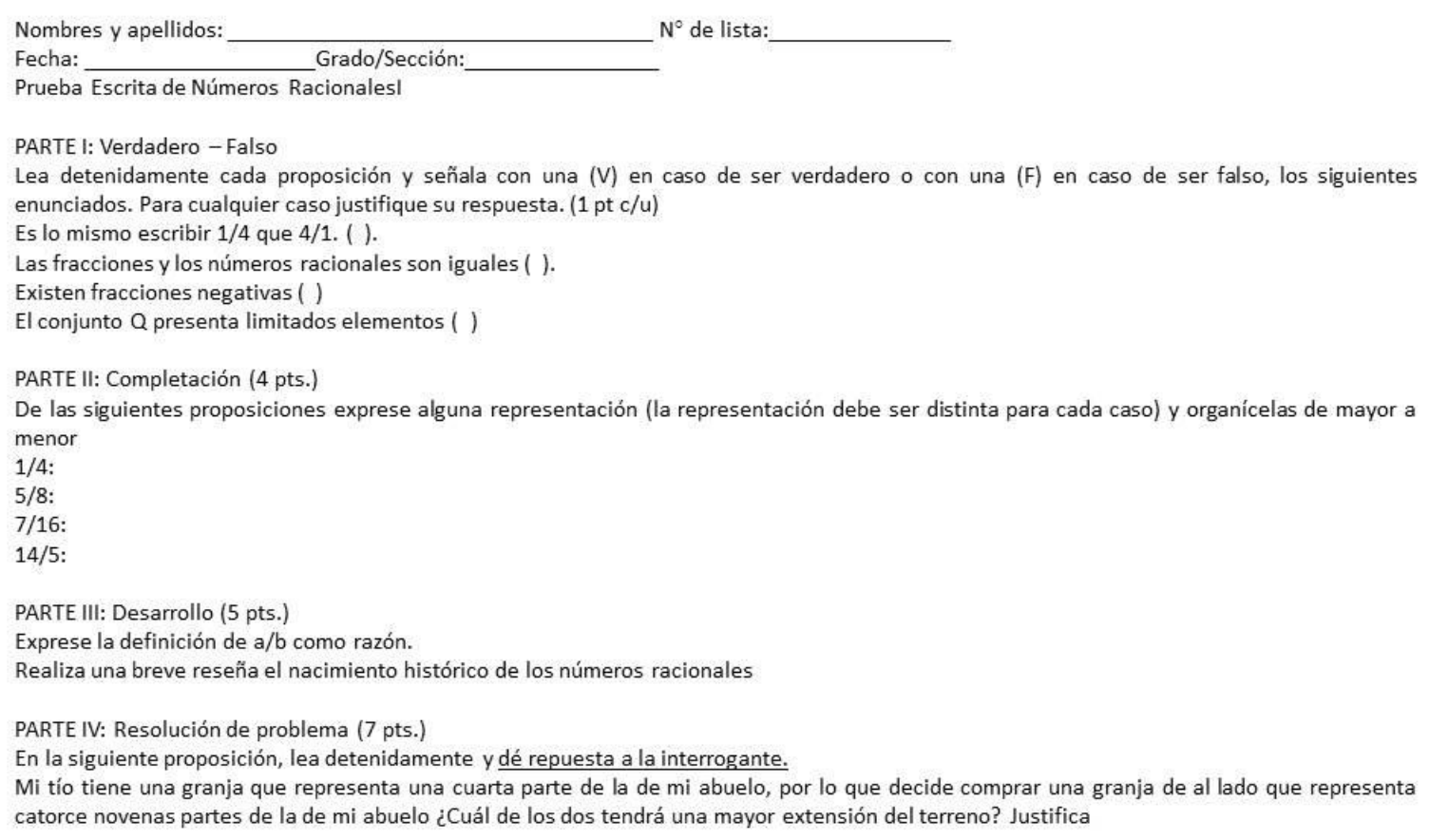

\section{Figura 1. Modelo de prueba}

A partir de la presentación del ejemplo de evaluación, el formador de profesores preguntó si el problema planteado podía ser un ejemplo o no de la conexión de las matemáticas con la realidad. Esta pregunta inicial dio lugar a un conjunto de intervenciones que ubicaron a los participantes a favor o en contra. La discusión coincidió con los planteamientos que se han hecho en el campo de la didáctica de las matemáticas sobre este tipo de problemas, es decir, la discusión se separó entre aquellos que consideraban al problema como "real" y aquellos que manifestaban que el hecho de hablar de un terreno no significaba que aquel planteamiento fuese real.

El formador en estas intervenciones mostró dominio de ese tipo de discusión acerca de si un problema es o no real. Había profesores que manifestaban que el problema era "real" porque contenía enunciados que hacían referencia a una mínima información extramatemática ("Mi tío tiene una granja", “comprar una granja al lado",...), otros, por el contrario manifestaron que no lo era, sobre todo porque no les parecía real el uso de expresiones como "comprar una granja de al lado que representa catorce novenas partes de la de mi abuelo". La dirección de este debate llevada por el formador permitió incorporar términos como "problema auténtico", "problema real", "problema situado" que, a decir de Beswick (2011) reflejan diferentes grados de distinción de la realidad en la presentación de los problemas matemáticos.

La adecuada intervención del formador en cuanto a mostrar un dominio del conocimiento pedagógico en un contexto matemático, más la conducción que realizó planteando preguntas, ejemplos y contraejemplos que desencadenaran una discusión, son la muestra de que la 
problematización es un recurso metodológico adecuado para la formación de profesores de matemáticas.

\section{Reflexiones Finales}

En lo expuesto se ha visto que el conocimiento especializado que debe poseer todo formador de profesores consigue en la problematización un modo privilegiado para aplicarlo en sus espacios formativos. La conjugación de ambos permite promover una formación caracterizada por la reflexión sobre la práctica educativa matemática.

Esta complementariedad de la problematización con los conocimientos del formador de profesores de matemáticas, da respuesta a la necesidad que se tiene de implementar procesos formativos que permitan egresar de nuestras instituciones profesores de matemáticas que respondan a las demandas laborales y sociales que se les plantean. Esto se afirma porque es común que muchos recién egresados como profesores se percaten que gran parte de lo que estudiaron en las aulas para ser profesor no responde a las demandas sociales y laborales que se le hacen. Esta sensación de no ver el sentido a muchos de los cursos que vieron en su formación obedece a que estos no se vincularon con la reflexión sobre la práctica educativa.

A través de la problematización de la práctica educativa matemática se contextualizan tanto la matemática como la pedagogía que debe conocer el estudiante para profesor, dándole sentido a los diferentes conocimientos que se imparten en las instituciones de formación de profesores.

Sin embargo queda aún mucho camino por recorrer. En el caso de la problematización como metodología de trabajo para fortalecer los procesos formativos de profesores aún hay muchas aristas desconocidas; por ejemplo, no se conoce con exactitud hasta qué punto la toma de conciencia a partir de la problematización permanece en el tiempo. Tampoco está claro qué tanto las creencias personales del formador como la de los que se forman, afectan el proceso de problematización o no.

En el caso del formador de profesores también hay muchas interrogantes. La parte afectiva, la manera como enfrenta los imprevistos en el aula o la forma como estos conocimientos se articulan con la investigación, son campos aún muy desconocidos

Estos aciertos y estas dudas son las que animan a continuar el estudio del formador de profesores y la problematización. La problematización es un camino aun largo de recorrer y el del formador de profesores, también. Queda continuar con esta empresa que todavía tiene mucho que avanzar si queremos ir mejorando los procesos formativos de los profesores, aspecto clave en la calidad educativa.

\section{Referencias}

Baez Melendres, Mayra; Farfán Márquez, Rosa María (2017) Reflexionar sobre la matemática escolar. Una ruta socioepistemológica. Acta Latinoamericana de Matemática Educativa Vol. 30, pp. 1037-1045. 
Ball, D. L. , Sleep, L., Boerst, T., \& Bass, H. (2009). Combining the development of practice and the practice of development in teacher education. Elementary School Journal. Vol. 109 ,pp. 458-476.

Ball, Deborah Loewenberg; Thames, Mark Hoover and Phelps, Geoffrey (2008) Content Knowledge for Teaching: What Makes It Special? Journal of Teacher Education. Vol. 59 (5), pp. 389-407

Beswick, Kim (2011) Teachers' beliefs about school mathematics and mathematicians' mathematics and their relationship to practice. Educational Studies in Mathematics. Vol. 79 (1), pp. 127-147

Bless Gutieerez, Victor; Diaz Columbié, Yamicela (2010). La problematización de las ciencias escolares como desafío posible: enfoque a una educación científica de calidad desde la concepción científica del aprendizaje basado en problemas. Cuadernos de Educación y Desarrollo. Vol. 2 (22). Recuperado de http://www.eumed.net/rev/ced/index.htm

Borjas, Beatriz; Ortiz, Marielsa; Rodríguez, Elvis; Soto, María Cristina (2015) La formación de educadoras y educadores populares. Una propuesta para la transformación de las prácticas. Colombia. Federación Internacional de Fe y Alegría

Cantoral, Ricardo; Montiel, Gisela; Reyes-Gasperini, Daniela (2015). El programa socioepistemológico de investigación en matemática educativa: el caso de Latinoamérica. Revista Latinoamericana de Investigación en Matemática Educativa. Vol. 18 (1), pp.5-17. DOI: $10.12802 /$ relime.13.1810

Carrillo, J.; Climent,N.; Contreras,L.C.; Muñoz-Catalán,M. C. Mathematics Teacher Specialized Knowledge. In: Proceedings of the Eighth Congress of the European Society for Research in Mmathematics, 8th, 2013, Antalya. CERME 8, Antalya, Turquía: CERME 8, pp. 2985-2994.

Chapman, O. (2011). The field of research in mathematics teacher education. Journal of Mathematics Teacher Education. Vol. 14, pp. 247-249.

Chick, Helen \& Beswick, Kim (2017) Teaching teachers to teach Boris: a framework for mathematics teacher educator pedagogical content knowledge. Springer

Dewey (1920) citado por Fabre, Michael (2006) Qu'est-ce que problématiser ? L'apport de John Dewey en Fabre, Michael et Vellas, Etiennette (éditeurs) Situations de formation et problématisation. Édition de Boecks Université. France. ISBN 2-8041-5245-6 pp. 17-30

Dewey, Jhon (1993). Logique, la théorie de l'enquête. France. PUF

Diaz Marsá, Marcos (2008) ¿Qué quiere decir pensar? Acerca de la noción de problematización en Michel Foucault. Revista de Filosofía. Vol 43, pp.51-70

Fabre, Michael (2005) Qu'est-ce que problématiser ? L’apport de John Dewey en Fabre, Michael et Vellas, Etiennette (éditeurs) Situations de formation et problématisation. ISBN 2-8041-5245-6 pp. 17-30 France. Édition de Boecks Université

Fabre, Michel ; Musquer, Agnés (2009) Les inducteurs de problématisation. Les Sciences de l'éducation - Pour l'Ére nouvelle. 42 (3) : 11-129. Recuperado de https://www.cairn.info/revue-les-sciences-de-l-education-pour-l-erenouvelle-2009-3-page$\underline{111 . h t m}$ 
Foucault, Michel.(1984) Polémica, política y problematizaciones, en Estética, ética y hermenéutica. Obras Esenciales. III ( 353-361). España. Paidós.

Freire, Paulo (2002). Concientización: Teoría y práctica de una educación liberadora. Buenos Aires. Galerna

Freire, Paulo. (1970). Pedagogía del oprimido. México: Siglo XXI

Godino, J. D.; Giacomone, B.; Font, V. y Pino-Fan, L. (2018). Conocimientos profesionales en el diseño y gestión de una clase sobre semejanza de triángulos. Análisis con herramientas del modelo CCDM. Avances de Investigación en Educación Matemática. No. 13, pp. 63 83.

González-Vallejos, María Paz (2018). El estudio del formador latinoamericano: un campo de investigación 'en construcción'. magis, Revista Internacional de Investigación en Educación. Vol. 10 (21), pp. 35-54. DOI: 10.11144/Javeriana.m10-21.eflc

Ivars, Pere; Fernández, Ceneida; Llinares, Salvador (2016) Cómo estudiantes para maestros miran de manera estructurada la enseñanza de las matemáticas al escribir narrativas. La matematica e la sua didattica. Vol. 24 (1-2), pp. 79-96

Kosik, Karel (1967) Dialéctica de lo concreto. Estudio sobre los problemas del hombre y del mundo. México. Editorial Grijalbo

Llinares, Salvador (2013). El desarrollo de la competencia docente "mirar profesionalmente" la enseñanza- aprendizaje de las matemáticas. Educar em. Vol. 50, pp. 117-133 Recuperado de: http://www.redalyc.org/articulo.oa

Organización de las Naciones Unidas para la Educación, la Ciencia y la Cultura, UNESCO (2016). Estrategia regional sobre docentes: Estado del arte y criterios orientadores para la elaboración de políticas de formación y desarrollo profesional de docentes de primera infancia en América Latina y el Caribe. Santiago de Chile: Oficina Regional de Educación para América Latina y el Caribe de la Organización de las Naciones Unidas para la Educación, la Ciencia y la Cultura, OREALC-UNESCO. Disponible en: http://unesdoc.unesco.org/ images/0024/002451/245157s.pdf

Parra-Sandoval y Villa-Ochoa, Jhony Alexander (2017) Vinculación de las matemáticas con la realidad. Implicaciones en la conformación del pensamiento profesional del docente. Paradigma Vol. XXXVIII, $N^{o}$ Extraordinario, pp. 288-312. Disponible en http://revistas.upel.edu.ve/index.php/paradigma/issue/view/448

Peña-Rincón, Pilar-Alejandra, Hueitra-Santibañez, Yolanda Conocimientos [matemáticos] mapuche1 desde la perspectiva de los educadores tradicionales de la comuna de El Bosque. Revista Latinoamericana de Etnomatemática [en linea]. 2016, 9(1), 8-25[fecha de Consulta 20 de Abril de 2020]. ISSN: . Disponible en: https://www.redalyc.org/articulo.oa?id=274044103002

Rodríguez Arocho, Wanda (2015) Reflexividad histórica, problematización e indagación dialógica como herramientas para repensar el concepto vygotskiano de zona de desarrollo próximo. Revista Puertoriqueña de Psicología. Vol. 26 (1), pp. 10-24 
Rowland, T., Huckstep, P., \& Thwaites, A. (2005). Elementary teachers' mathematics subject knowledge: The knowledge quartet and the case of Naomi. Journal of Mathematics Teacher Education. Vol. 8, pp. 255-281.

Sánchez García, Victoria; García Blanco, Mercedes (2004) Formadores de profesores de matemáticas. Una aproximación teórica a su conocimiento profesional. Revista de Educación. No. 333, pp. 481-493

Shön, Donal (1998). El profesional reflexivo. Cómo piensan los profesionales cuando actúan. Barcelona: Paidós.

Zaslavsky, O., \& Leikin, R. (2004). Professional development of mathematics teacher educators: Growth through practice. Journal of Mathematics Teacher Education. No. 7, pp. $5-32$. 